\title{
Urinary transglutaminase 2 as a potent biomarker to predict interstitial fibrosis and tubular atrophy of kidney allograft during early posttransplant period in deceased donor kidney transplantation
}

\author{
Jee Yeon Kim¹, Yu-Mee Wee', Monica Young Choi', Hey Rim Jung', Ji Yoon Choi', Hyun Wook Kwon', \\ Joo Hee Jung', Yong Mee Cho², Heounjeong Go², Minkyu Han ${ }^{3}$, Young Hoon Kim ${ }^{1}$, Duck Jong Han', Sung Shin \\ ${ }^{1}$ Division of Kidney and Pancreas Transplantation, Department of Surgery, Asan Medical Center, University of Ulsan College of \\ Medicine, Seoul, Korea \\ ${ }^{2}$ Department of Pathology, Asan Medical Center, University of Ulsan College of Medicine, Seoul, Korea \\ ${ }^{3}$ Department of Clinical Epidemiology and Biostatistics, Asan Medical Center, Seoul, Korea
}

\begin{abstract}
Purpose: Transglutaminase type 2 (TG2) is an extracellular matrix crosslinking enzyme with a pivotal role in kidney fibrosis. We tested whether quantification of urinary TG2 may represent a noninvasive method to estimate the severity of kidney allograft fibrosis.

Methods: We prospectively collected urine specimens from 18 deceased donor kidney transplant recipients at 1-day, 7-day, 1-month, 3-month, and 6-month posttransplant. In addition, kidney allograft tissue specimens at 0-day and 6-month posttransplant were sampled to analyze the correlation of urinary TG2 and kidney allograft fibrosis.

Results: Thirteen recipients had increased interstitial fibrosis and tubular atrophy (IFTA) scores at the 6-month protocol biopsy (IFTA group). The mean level of urinary TG2 in the IFTA group was higher compared to that of 5 other recipients without IFTA (no IFTA group). Conversely, the mean level of urinary syndecan-4 in the IFTA group was lower than levels in patients without IFTA. In the IFTA group, double immunofluorescent staining revealed that TG2 intensity was significantly upregulated and colocalizations of TG2/heparin sulfate proteoglycan and nuclear syndecan-4 were prominent, usually around tubular structures.

Conclusion: Urinary TG2 in early posttransplant periods is a potent biomarker for kidney allograft inflammation or fibrosis.

[Ann Surg Treat Res 2019;97(1):27-35]
\end{abstract}

Key Words: Biomarkers, Transglutaminase 2, Kidney transplantation

\section{INTRODUCTION}

Kidney transplantation is considered the best treatment option available for patients with end-stage renal disease. However, rejection and fibrosis are leading causes of allograft failure. Interstitial fibrosis and tubular atrophy (IFTA), previously known as chronic allograft nephropathy (CAN), is currently diagnosed by pathologic changes involving all parts of the renal parenchyma. It is critical to distinguish IFTA from acute rejection for optimal clinical decision-making following
Received March 6, 2019, Revised April 26, 2019,

Accepted May 14, 2019

Corresponding Author: Sung Shin

Division of Kidney and Pancreas Transplantation, Department of Surgery, Asan Medical Center, University of Ulsan College of Medicine, 88 Olympicro 43-gil, Songpa-gu, Seoul 05505, Korea

Tel: +82-2-3010-3964, Fax: +82-2-474-9027

E-mail: sshin@amc.seoul.kr

ORCID code: https://orcid.org/0000-0001-7318-4208
Copyright (c) 2019, the Korean Surgical Society

(c) Annals of Surgical Treatment and Research is an Open Access Journal. All articles are distributed under the terms of the Creative Commons Attribution NonCommercial License (http://creativecommons.org/licenses/by-nc/4.0/) which permits unrestricted non-commercial use, distribution, and reproduction in any medium, provided the original work is properly cited. 
kidney transplantation [1].

Needle biopsy as an invasive procedure has become safer, and biopsy interpretation more standardized [2]; however, it is still associated with risk of postbiopsy bleeding which can lead to graft loss, and sampling errors, and interobserver variability in interpretation remains problematic [3]. Moreover, interchangeable histology classifications are becoming more difficult because of continuous revisions of the histological diagnostic criteria every 2 years [4]. Noninvasive biomarkers are necessary to monitor allograft status and to predict long-term outcomes. Several candidates have been suggested as useful biomarkers for acute rejection or fibrosis of kidney allografts $[5,6]$.

Transglutaminase type 2 (TG2) is an extracellular matrix (ECM) crosslinking enzyme known to have a pivotal role in kidney fibrosis. The interaction of TG2 with heparin sulfate proteoglycan (HSPG) syndecan-4 (SDC4) regulates cell surface trafficking and localization [7]. Accumulation in the ECM is the histological hallmark of IFTA and is responsible for progressive tubulointerstitial fibrosis [8]. Here, we aim to investigate urinary TG2 as a noninvasive biomarker for prediction of fibrosis in kidney allografts following deceased donor kidney transplantation (DDKT).

\section{METHODS}

\section{Subjects}

From January 2015 to July 2016, patients aged 18 to 80 years old who received DDKT at our center were included in this study. Patients who needed multiorgan transplantation were excluded from this study. Patients requiring regular antiplatelet or anticoagulant treatments were also excluded. We prospectively collected urine specimens from 20 DDKT recipients at 1-day, 7-day, 1-month, 3-month, and 6-month posttransplant intervals. In addition, kidney allograft tissue specimens at 0 -day and 6-month posttransplant were sampled to analyze the correlation of urinary TG2 and kidney allograft fibrosis. Of the 20 patients who were enrolled before transplantation, 18 recipients were included in the final analysis because urine samples of 2 patients were not available posttransplant. This study received approval from the Institutional Review Board (IRB) of Asan Medical Center (approval number: 2014-1214) and all patients provided written informed consent.

\section{Endpoints}

The objective of this study was to analyze the correlation between urinary TG2 and the progression of allograft fibrosis between 0-day and 6-month protocol biopsies. The severity of allograft fibrosis was estimated by summing Banff ct, ci, and cv scores [4] of each biopsy specimen. Participants were categorized into 2 groups based on the progression of allograft fibrosis. Recipients were categorized as the IFTA group if the sum of ct, ci, and cv scores on 8-month protocol biopsy increased by one or more compared with the sum of the scores on the 0-day biopsy.

\section{Urine collection and tissue preparation}

Samples were collected under human subject research protocols approved by the IRB of Asan Medical Center. A urine sample was collected on posttransplant day 1, 7 days, 1 month, 3 months, and 6 months. About 35- to 50-mL urine sample was collected from each patient. For each urine specimen, 0.5 $\mathrm{mL}$ of a protease inhibitor mixture (5 mM 4-(2-aminoethyl) benzensulfonyl fluoride hydrolchloride, $0.2 \mathrm{mM}$ leupeptinhemisulfate, and $333 \mathrm{mM}$ sodium azide) was added. To remove urinary sediments including whole cells, large membrane particles, and other debris, urine specimens were centrifuged at $4,000 \mathrm{rpm}$ for 15 minutes at $4^{\circ} \mathrm{C}$. An aliquot of supernatant was stored at $-80^{\circ} \mathrm{C}$ until use.

Allograft protocol biopsies were obtained at implantation and by ultrasound-guided percutaneous puncture 6 months after transplantation. Histological diagnosis was performed based on tissue studies using light microscopy. All biopsy specimens were graded according to the Banff 2013 classification [4] by 2 renal pathologists.

\section{Urine protein profiling for urinary markers}

TG2 (Mybiosource, San Diego, CA, USA), SDC4 (R\&D System, Minneapolis, MN, USA), and $\alpha-1$ microglobulin (A1M) (Abcam, Cambridge, UK) levels were measured by an enzymelinked immunosorbent assay (ELISA) kit according to the manufacturer's protocol. Briefly, $100 \mu \mathrm{L}$ of 60 -fold diluted urine sample and standards were added to the bottom of a microELISA plate well before incubation for 90 minutes at $37^{\circ} \mathrm{C}$. A biotinylated detection antibody was then added to each well and incubated for 1 hour at $37^{\circ} \mathrm{C}$. The plate was washed 3 times with wash buffer. Horseradish peroxidase-conjugated working solution was then added to each well and was incubated for 30 minutes at $37^{\circ} \mathrm{C}$. Absorbance of each well was detected on a microplate reader (Sunrise, Tecan, Switzerland) set to 450 $\mathrm{nm}$. To detect A1M, $50 \mu \mathrm{L}$ of 20,000-fold diluted urine sample and standards were added to the well and were incubated for 120 minutes at room temperature. To detect SDC4, $100 \mu \mathrm{L}$ of nondiluted urine sample were added to the well and were incubated for 120 minutes at room temperature. Thereafter, procedures similar to TG2 ELISA indicated above were performed according to each kit's protocol.

The level of IL- 6 was measured with a cytometric bead array (CBA) method using the CBA flexset kit (BD Life Sciences, San Jose, CA, USA), according to the manufacturer's protocol. Briefly, $50 \mu \mathrm{L}$ of mixed capture beads were added to the same 
volume of urine or standards and incubated for 1 hour at room temperature in the dark with shaking to allow the cytokine to bind to the capture beads. PE detection reagent was then added to each tube and incubated for 2 hours at room temperature in the dark with shaking. We performed additional steps to enhance the signal, increasing enhancing reagent treatment by 1 hour. The fluorescence-labeled cytokine-specific beads were analyzed by flow cytometry and assessed using FLAP array software (BD Life Sciences).

\section{Immunofluorescence and confocal microscopy}

Kidney allograft biopsy tissue samples were cryosectioned, and immunolabeled following collection procedures. Samples were placed into cryomolds with optimal cutting temperature medium and frozen over liquid nitrogen. Thin sections (4to $6-\mu \mathrm{m}$ thickness) obtained via cryosectioning were labeled with antibodies against TG2, SDC4, HSPG (Abcam) and the nucleus was labeled with 4;,6-diamidino-2-phenylindole (DAPI; Sigma-Aldrich, St. Louis, MO, USA). Sections were incubated with primary antibodies for 16 hours at $4^{\circ} \mathrm{C}$, and washed twice in phosphate buffered saline containing $0.5 \%$ bovine serum albumin. Next, fluorescence conjugated secondary antibodies (goat anti-rat or anti-rabbit IgG antibodies conjugated with AlexaFluor488 or AlexzFluor555, Life Technology, Carlsbad, CA, USA) were applied for staining and sections were incubated with DAPI for 5 minutes. Confocal imaging was performed using a laser scanning confocal microscope equipped with a hybrid detector (LSM710, Carl Zeiss. Germany).

Individual fluorophores were imaged sequentially with the excitation wavelength switching at the end of each frame. Images were analyzed using custom software written in ZEN (Zen 2 blue edition, Carl Zeiss Microscopy GmbH, Jena, Germany). Data are expressed as mean fluorescence intensities (MFI). Geometric properties for colocalization position and area were calculated as identified. For each TG2 and SDC4 cluster, a list of HSPG and nucleus clusters were compiled, which included directly overlapping clusters.

\section{Statistical analysis}

Differences among values were analyzed using the chi-square

Table 1. Recipient and donor characteristics of the participants

\begin{tabular}{|c|c|c|c|c|}
\hline Characteristic & All $(n=18)$ & IFTA $(n=13)$ & No IFTA $(n=5)$ & P-value \\
\hline \multicolumn{5}{|l|}{ Recipient } \\
\hline Age $(y r)$ & $52.4 \pm 10.6$ & $46.6 \pm 11.3$ & $62.0 \pm 3.3$ & 0.128 \\
\hline Female sex & $9(50.0)$ & $6(46.2)$ & $3(60.0)$ & $>0.999$ \\
\hline $\mathrm{BMI}\left(\mathrm{kg} / \mathrm{m}^{2}\right)$ & $22.6 \pm 2.4$ & $22.4 \pm 2.7$ & $23.4 \pm 1.3$ & 0.436 \\
\hline Cause of ESRD & & & & 0.584 \\
\hline Hypertension & $3(16.7)$ & $2(15.4)$ & $1(20.0)$ & \\
\hline Diabetes & $3(16.7)$ & $1(7.7)$ & $2(40.0)$ & \\
\hline Glomerulonephritis & $2(11.1)$ & $2(15.4)$ & 0 & \\
\hline IgA nephropathy & $2(11.1)$ & $2(15.4)$ & 0 & \\
\hline FSGS & $1(5.6)$ & $1(7.7)$ & 0 & \\
\hline Unknown/others & 7 (58.9) & $5(38.5)$ & $2(40.0)$ & \\
\hline Duration of pretransplant dialysis (mo) & $94(12-172)$ & $94(15-172)$ & $93(12-138)$ & 0.967 \\
\hline Induction therapy & & & & 0.521 \\
\hline Thymoglobuline & $3(16.7)$ & $3(23.1)$ & 0 & \\
\hline Simulect & $15(83.3)$ & $10(76.9)$ & $5(100.0)$ & \\
\hline Delayed graft function & $3(16.7)$ & $2(15.4)$ & $1(20.0)$ & $>0.999$ \\
\hline \multicolumn{5}{|l|}{ Donor } \\
\hline Female sex & $3(16.7)$ & $2(15.4)$ & $1(20.0)$ & $>0.999$ \\
\hline $\mathrm{BMI}\left(\mathrm{kg} / \mathrm{m}^{2}\right)$ & $22.9 \pm 3.1$ & $22.5 \pm 3.3$ & $24.1 \pm 2.7$ & 0.337 \\
\hline Cold ischemic time (hr) & $272 \pm 96$ & $284 \pm 108$ & $241 \pm 53$ & 0.422 \\
\hline ECD & $3(16.7)$ & $3(23.1)$ & $0(0)$ & 0.523 \\
\hline Brain death by CVA & $6(33.3)$ & $6(46.2)$ & $0(0)$ & 0.107 \\
\hline Terminal serum creatinine $(\mathrm{mg} / \mathrm{dL})$ & $1.78 \pm 1.66$ & $1.97 \pm 1.78$ & $1.28 \pm 1.31$ & 0.443 \\
\hline $\mathrm{PRA}>50 \%$ & $3(16.7)$ & $2(15.4)$ & $1(20.0)$ & $>0.999$ \\
\hline HLA mismatch & $2.6 \pm 2.1$ & $2.8 \pm 2.2$ & $2.2 \pm 1.8$ & 0.615 \\
\hline DSA positive & $2(11.1)$ & $2(15.4)$ & $0(0)$ & $>0.999$ \\
\hline
\end{tabular}

Values are presented as mean \pm standard deviation, number $(\%)$, or median (range).

IFTA, interstitial fibrosis and tubular atrophy; BMI, body mass index; ESRD, end-stage renal disease; FSGS, focal segmental glomerulosclerosis; ECD, expanded criteria donor; CVA, cerebrovascular accident; PRA, panel-reactive antibody; HLA, human leukocyte antigen; DSA, donor-specific antibody. 
Table 2. Comparison of histologic findings of protocol biopsies of the IFTA and no IFTA groups

\begin{tabular}{lccccc}
\hline \multirow{2}{*}{$\begin{array}{c}\text { Banff } \\
\text { score }\end{array}$} & \multicolumn{2}{c}{ IFTA $(\mathrm{n}=13)$} & \multicolumn{2}{c}{ No IFTA $(\mathrm{n}=5)$} \\
\cline { 5 - 6 } & Zero day & 6 Months & & Zero day & 6 Months \\
\hline $\mathrm{g}$ & 0 & $0.3 \pm 0.5$ & 0 & $0.4 \pm 0.5$ \\
$\mathrm{i}$ & $0.1 \pm 0.3$ & $0.3 \pm 0.6$ & $0.4 \pm 0.9$ & 0 \\
$\mathrm{t}$ & 0 & $0.8 \pm 0.9$ & $0.2 \pm 0.4$ & $0.8 \pm 1.3$ \\
$\mathrm{v}$ & 0 & 0 & 0 & 0 \\
$\mathrm{cg}$ & 0 & 0 & 0 & $0.2 \pm 0.4$ \\
$\mathrm{ci}$ & $0.4 \pm 0.7$ & $1.6 \pm 0.7$ & $0.4 \pm 0.5$ & 0 \\
$\mathrm{ct}$ & $0.6 \pm 0.7$ & $1.0 \pm 0.4$ & $0.6 \pm 0.05$ & $0.4 \pm 0.5$ \\
$\mathrm{cv}$ & $0.2 \pm 0.4$ & $1.2 \pm 0.8$ & $0.2 \pm 0.4$ & $0.2 \pm 0.4$ \\
$\mathrm{~mm}$ & 0 & $0.1 \pm 0.3$ & 0 & $0.2 \pm 0.4$ \\
$\mathrm{ah}$ & $0.2 \pm 0.6$ & $1.2 \pm 1.2$ & $0.2 \pm 0.4$ & $0.6 \pm 1.3$ \\
$\mathrm{ptc}$ & 0 & 0 & 0 & 0
\end{tabular}

IFTA, interstitial fibrosis and tubular atrophy.

A

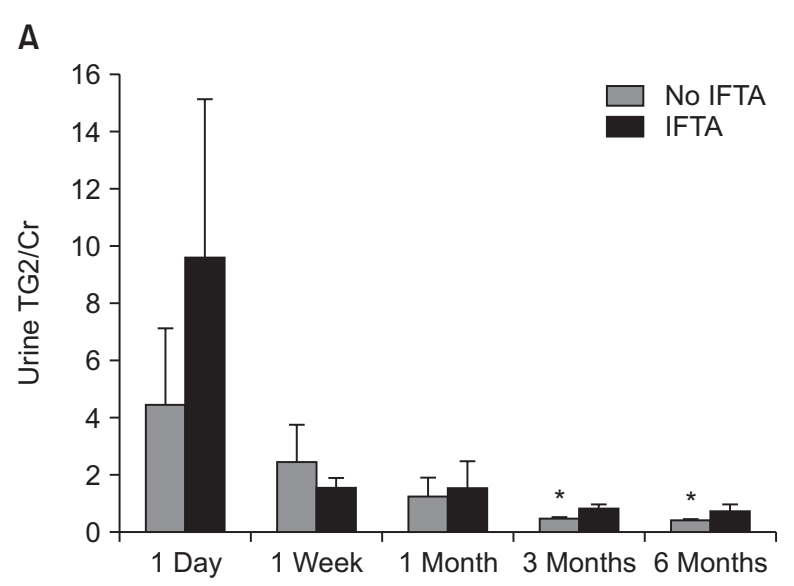

C

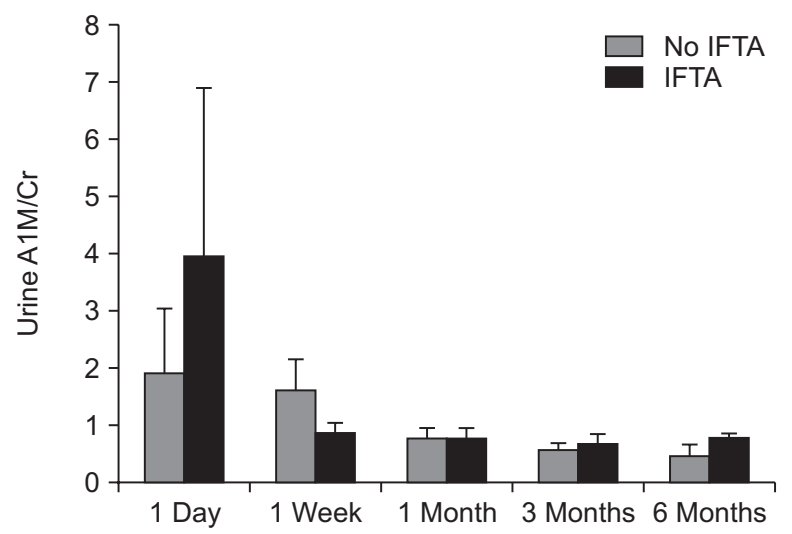

test, Fisher exact test, Mann-Whitney, t-tests or paired t-tests, as applicable.

All statistical analyses were performed using IBM SPSS Statistics ver. 20.0 (IBM Co., Armonk, NY, USA) and R ver. 3.1.2 (R Foundation for Statistical Computing, Vienna, Austria). A P-value of $<0.05$ was considered to be statistically significant.

\section{RESULTS}

\section{Recipient and donor characteristics and transplantation}

We prospectively collected urine samples and kidney biopsy specimens from 20 recipients that underwent DDKT at our center between January 2015 and July 2016. Two patients whose urine samples were not available were excluded; thus, 18 recipients were included in this study. Based on the progression of allograft fibrosis, participants were divided into 2 groups: the IFTA and no IFTA groups. Baseline characteristics of participants are shown in Table 1. Among the 18 patients, only

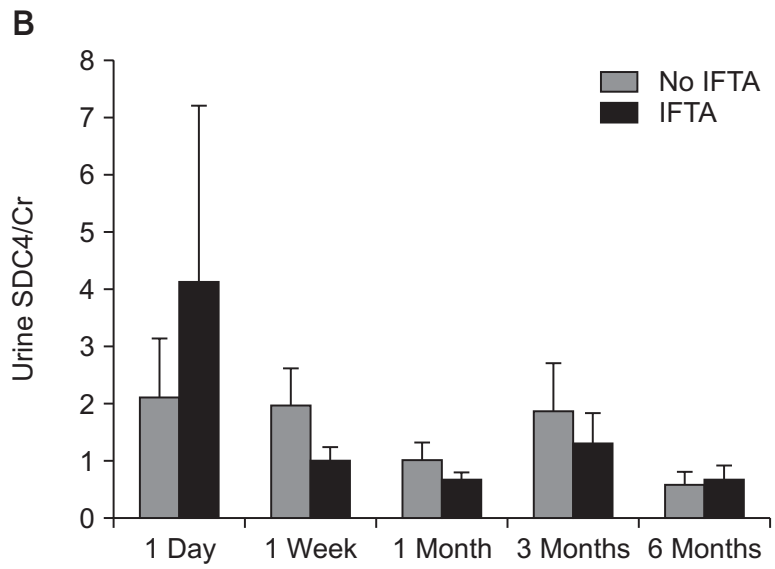

Fig. 1. Urinary biomarkers after adjusted for creatinine $(\mathrm{mg} / \mathrm{mL})$ normalization in the interstitial fibrosis and tubular atrophy (IFTA) and no IFTA groups. (A) The level of urinary transglutaminase 2 (TG2) $(\mathrm{ng} / \mathrm{mL})$ in the IFTA group was significantly increased compared with the no IFTA group at 3- and 6-month posttransplant follow-up periods. (B-D) The level of urinary syndecan-4 (SDC4) (pg/mL), $\alpha-1$ microglobulin $(A 1 M)(\mathrm{ng} / \mathrm{mL})$, and IL-6 (fg/mL) between the 2 groups showed no significant differences. Cr, creatinine. 
1 required retransplantation. Three patients received rabbit antithymocyte globulin (Thymoglobulin, Genzyme, Cambridge, MA, USA), whereas the others received anti-IL-2 receptor antibody (Basiliximab) as an induction regimen. All patients received triple therapy as maintenance immunosuppression at the time of discharge; a combination of a calcineurin inhibitors, a mycophenolic acid derivative, and steroids. The target trough level for tacrolimus was $5-8 \mathrm{ng} / \mathrm{mL}$ during the early postoperative period. One patient in no IFTA group with underlying diabetes was administered cyclosporin with the target trough level $100-150 \mu \mathrm{g} / \mathrm{L}$ during the early postoperative period. Three recipients experienced delayed graft function after DDKT and there was no significant difference between the 2 groups. Three donors were defined expanded criteria donors according to the criteria proposed by the United Network for Organ Sharing. There was no significant difference in baseline characteristics between the 2 groups.

\section{A}

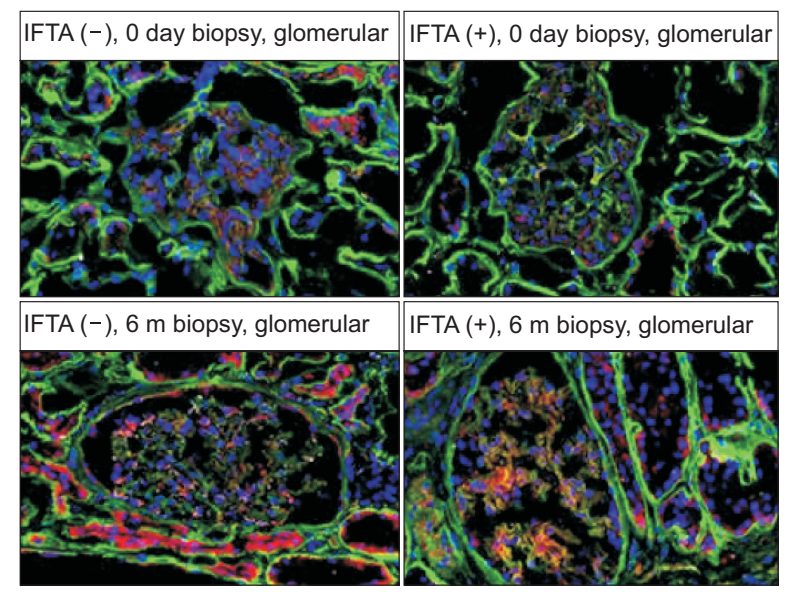

\section{C}

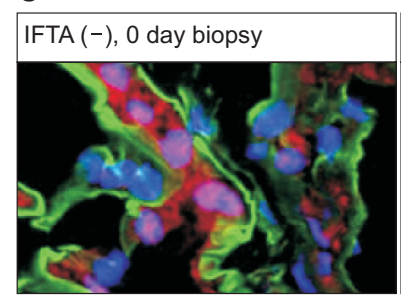

IFTA (-), $6 \mathrm{~m}$ biopsy, glomerular

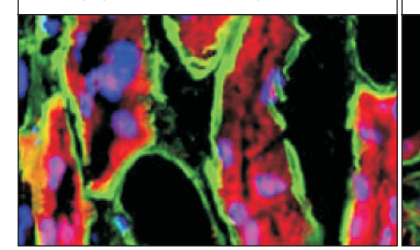

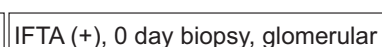

FTA (+), 0 day biopsy, glomerular

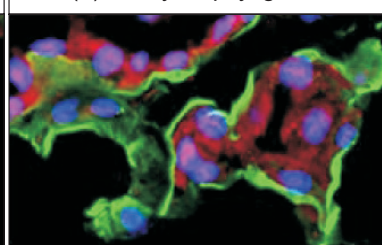

IFTA (+), 6 m biopsy, glomerular

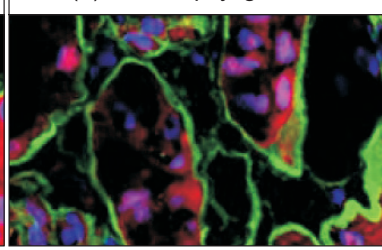

\section{Protocol biopsies of IFTA and no IFTA groups}

All patients had protocol biopsy at the time of transplantation (zero day) and at 6-month posttransplantation (Table 2). All patients had normal range serum creatinine and no recipient required for-cause biopsy until protocol biopsy was taken 6-month posttransplant. There was no significant difference in serum creatinine $(1.17 \pm 0.18$ in IFTA vs. $0.98 \pm 0.23$ in No IFTA, $P=0.173$ ) and estimated glomerular filtration rate value $(72.0 \pm 9.1$ in IFTA vs. $77.2 \pm 19.5$ in no IFTA, $P=1.0)$ using chronic kidney disease (CKD) epidemiology collaboration 2009 equation [9] at 6-month posttransplant between the 2 groups.

\section{Comparison of urinary biomarkers in IFTA and no IFTA group}

We compared the levels of urinary biomarkers between IFTA and no IFTA groups (Fig. 1). The level of urinary TG2 in the IFTA group was significantly increased compared with the no IFTA

\section{B}

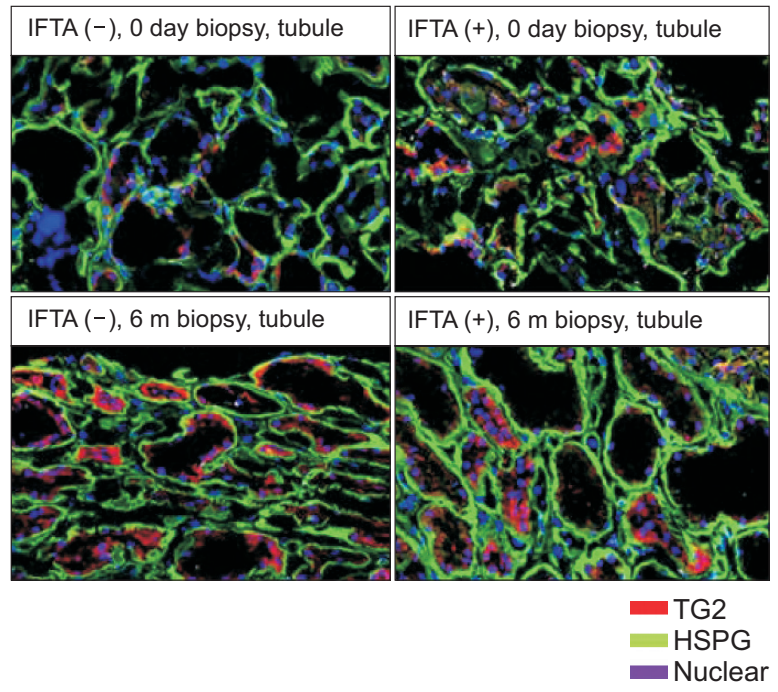

Fig. 2. Double immunofluorescence staining and confocal microscopy in representative allograft biopsy specimens in the interstitial fibrosis and tubular atrophy (IFTA) and no IFTA groups. (A) Transglutaminase 2 (TG2) intensity was significantly upregulated at the 6-month posttransplantation biopsies compared to those at 0-day posttransplantation. (B) TG2 intensity upregulation was prominent especially in tubular structures. (C) Colocalization of syndecan-4 and the nucleus was seen in biopsy specimens. HSPG, heparin sulfate proteoglycan. 
A

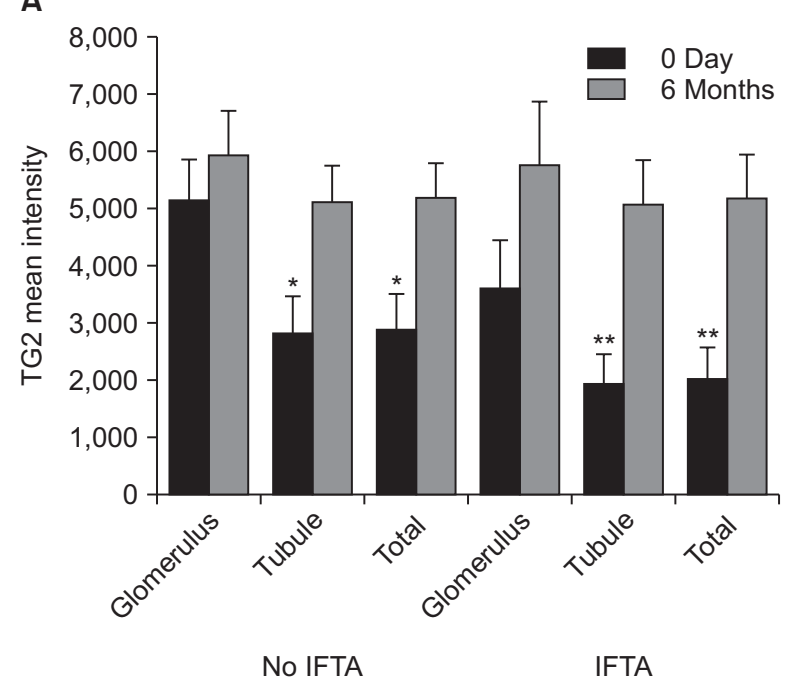

C

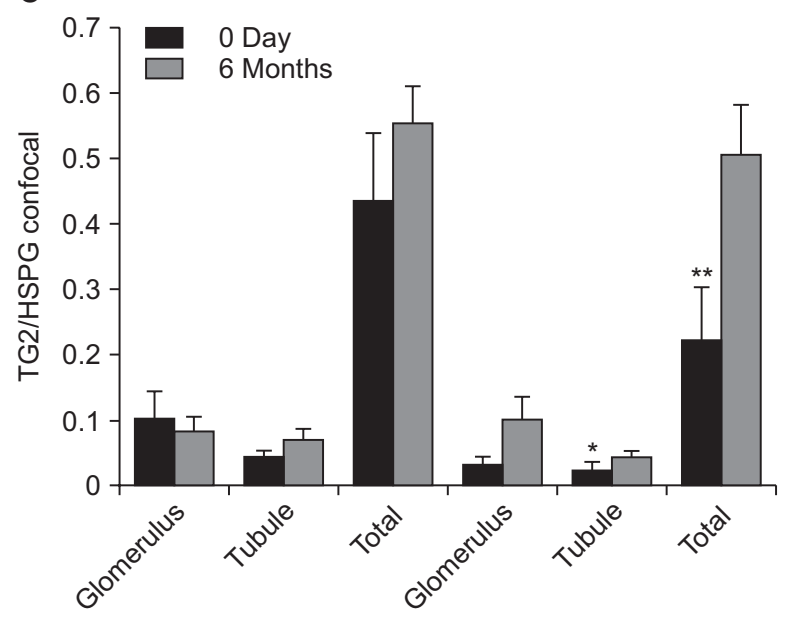

No IFTA

IFTA

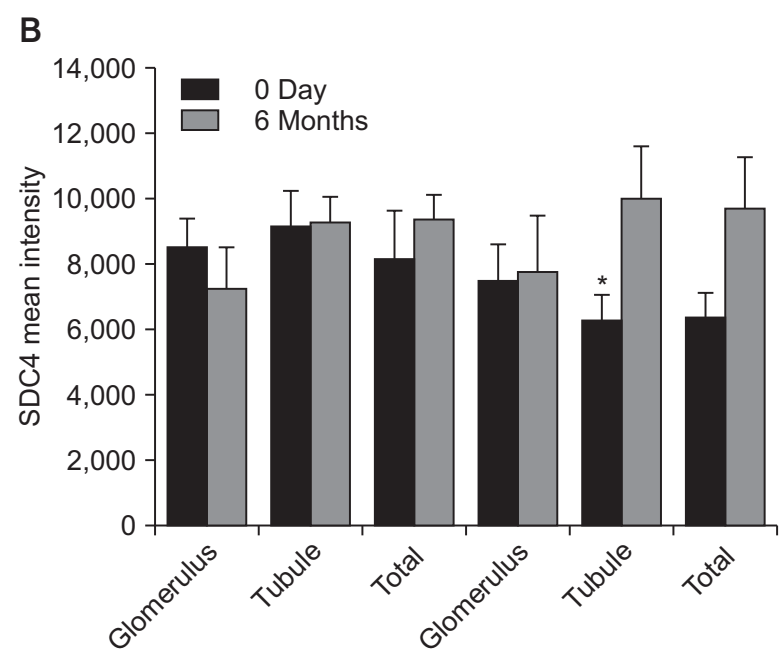

No IFTA

IFTA

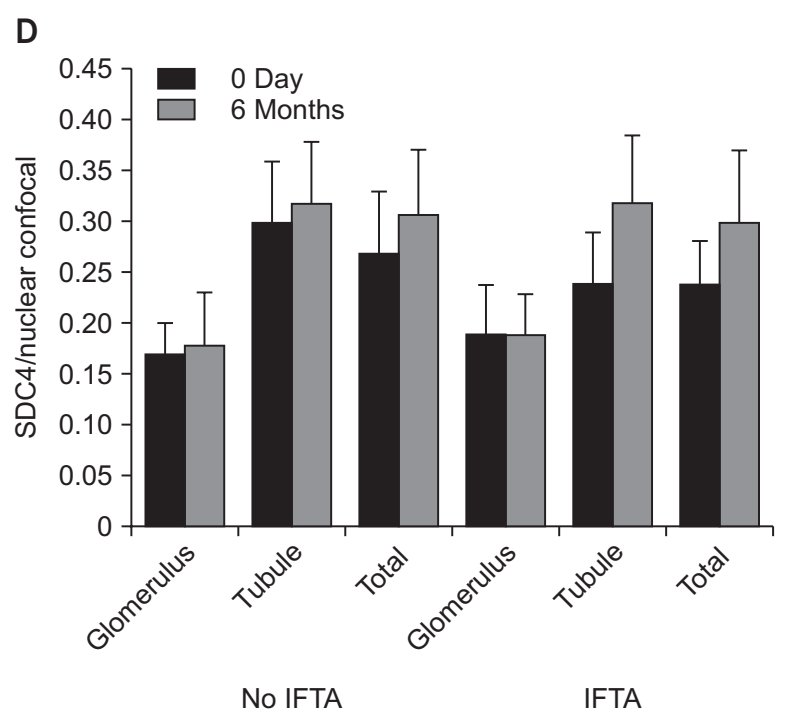

Fig. 3. (A, B) Intensity of transglutaminase 2 (TG2) and syndecan-4 (SDC4) in 0-day and 6-month biopsy specimens in the interstitial fibrosis and tubular atrophy (IFTA) and no IFTA groups in kidney structures. (C, D) Confocal microscopy intensity of TG2/heparan sulfate proteoglycan (HSPG) and SDC4/nuclear colocalization.

group at the 3-and 6-month posttransplant follow-up periods (Fig. 1A). There were no significant differences in the levels of urinary SDC4, A1M, and IL-6 between the 2 groups (Fig. 1B-D).

\section{Comparison of immunofluorescence staining of protocol biopsy specimens between the IFTA and no IFTA groups}

We performed double immunofluorescence staining in representative allograft biopsy specimens in the IFTA and no IFTA groups (Fig. 2). We also measured the intensity of TG2 and SDC4 in each individual specimen (Fig. 3). TG2 intensity was significantly upregulated at the 6-month posttransplantation biopsy compared to that of the 0 -day posttransplantation. This was prominent especially in tubular structures. The TG2
MFI difference was more remarkable $(P=0.006)$ in the IFTA group with a total intensity measuring 5,173 $\pm 784 \mathrm{MFI}$ at the 6-month biopsy compared to 2,035 $\pm 514 \mathrm{MFI}$ at the 0 -day $(5,223$ \pm 602 vs. $2,929 \pm 584$ in 6 months no IFTA group biopsies, $P=$ $0.01)$ (Fig. 3A).

While there was no change in SDC4 intensity between 0-day and 6-month biopsy samples in the no IFTA group, SDC4 was upregulated at 6 months compared to day 0 posttransplantation in the IFTA group and was prominent in tubular structures $(10,175 \pm 1,461$ vs. 6,366 \pm 711 , respectively, $\mathrm{P}=0.05)$ (Fig. 3B).

Colocalization of TG2 and HSPG was observed on confocal microscopy. No remarkable increase in colocalization of TG2 and HSPG was detected in the no IFTA group. TG2 and HSPG colocalization became prominent at the 6-month biopsy 
compared to the 0 -day biopsy in the IFTA group allograft specimens $(0.51 \pm 0.07$ vs. $0.23 \pm 0.08 \mathrm{MFI}$, respectively, $\mathrm{P}=$ 0.009 ) and this tendency was observed both in the glomerulus and the tubular structures (Fig. 3C). Although colocalization of SDC4 and the nucleus was also observed in biopsy specimens, confocal microscopy intensity showed no significant difference between the 6-month and 0-day biopsies for all structures in both groups (Figs. 2C and 3D).

\section{DISCUSSION}

Currently, there is a growing body of literature demonstrating that kidney tubule health can be measured noninvasively to provide insight into kidney graft function and disease progression beyond that provided by glomerular status [10]. Clinical chemistry and biochemistry diagnostics in transplantation relies on a limited set of biomarkers and a single molecular marker will likely never be identified that captures the function of a transplant organ in all its complexity [5].

As for urinary protein biomarkers, several studies have demonstrated promising candidates for injury and fibrosis of kidney allograft and association with CKD progression. In a prior FAVORIT trial analysis, neutrophil gelatinase-associated lipocalin, kidney injury molecule 1, IL-18, and liver-type fatty acid binding protein have been proposed as urinary biomarkers of kidney tubule injury or fibrosis [11] and these biomarkers have been proposed to measure kidney fibrosis status in DDKT [12]. Previous studies with biomarkers such as A1M, monocyte chemoattractant protein-1 (MCP-1), and type III N-terminal amino peptide in kidney transplant recipients have found that higher urinary concentration of each biomarker has been associated with greater tubulointerstitial fibrosis on kidney biopsy $[13,14]$. In other studies, metalloproteinases (MMPs) which are physiologic regulators of renal ECM degradation have been investigated, and changes in MMPs expression or activities such as MMP2, MMP9, MMP7 have been suggested as potential candidates for the detection of renal allograft injury and correlation with IFTA [15]. Urinary chemokines CXC chemokine ligand 9 and 10 have also been tested in urinary messenger RNA, microRNA and protein levels as noninvasive biomarkers of kidney allograft function $[15,16]$.

TG2 is a member of the transglutaminase enzyme family, involved in a plethora of biological functions such as angiogenesis [17], apoptosis, cell differentiation [18], and wound healing [19]. The role of TG2 has been shown to be crucial to both the ECM expansion $[20,21]$ and transforming growth factor beta (TGF- $\beta$ ) 1 activation $[22,23]$ that underlies fibrotic remodeling. In fibrotic diseases such as renal, liver and pulmonary fibrosis, increased TG2 externalization and expression results in abundant crosslink formation, contributing to ECM accumulation [21,22]. Our previous report revealed that TG2 has a potential effect in the stabilization of the ECM by interaction with fibronectin and TGF- $\beta$ in human abdominal aortic aneurysm [24]. HSPG SDC4 is an important binding partner for extracellular TG2. Interaction of TG2 with the HSPG SDC4 regulates cell surface trafficking, localization, and activity of TG2 [25]. The high affinity interaction of extracellular TG2 with SDC4 is thought to maintain the activation of protein kinase $\mathrm{Ca}$, and is important for controlling integrin levels and clustering throughout the cell surface [25,26]. Scarpellini et al. [7] demonstrated that SDC4 is required for cell surface targeting of TG2 and the development of kidney fibrosis in CKD and progressive fibrosis is associated with increase in extracellular TG2 and TG activity in the tubulointerstitium. They also showed in a CKD mouse model that extracellular TG2 colocalized with SDC4 in the tubular interstitium and basement membrane, where TG2 also colocalized with heparan sulfate chains. Richardson et al. [27] analyzed HSPG localization in corneal fibroblasts and revealed that the nuclear localization of HSPG core proteins was greater in fibronectin cells and proposed that fibronectin-mediated nuclear localization of HSPG might play an important role in inducing biological responses and regulating nuclear function. We have previously reported composition of urinary TG2, SDC4, IP-10, and MCP-1 as potent biomarkers to distinguish IFTA from tubulointerstitial inflammation in kidney transplant recipients [28]. To our knowledge, this is the first study to verify the value of urinary TG2 as a biomarker for allograft fibrosis and IFTA in deceased donor kidney transplant patients.

In this study, we analyzed potential urinary biomarkers of 18 DDKT recipients and revealed that the urinary levels of TG2 tended to be higher and that of SDC4 tended to be lower when IFTA was aggravated at the 6-month allograft biopsy. Urine molecule concentration depends on several biological variables such as fluid intake, body composition, in addition to hepatic and renal functions [29]. Since urinary creatinine concentration serves as an indicator of urine dilution, we measured the level of urine creatinine and mathematically adjusted for creatinine normalization of TG2 and SDC4 by dividing each concentration by the urine creatinine level. Higher levels of TG2 in the IFTA group were more remarkable after 1-month posttransplant when TG2 was normalized by creatinine. It is known that TG2 is upregulated by lysed red blood cells [30]. During the firstmonth posttransplant period hematuria is common. It may interfere with urinary TG2 levels, and urinary TG2 may not accurately reflect potential fibrosis of the kidney allograft. SDC4 after creatinine normalization did not show a significant difference between IFTA and no IFTA groups at 1-month posttransplant. Notably, A1M, which has been identified as a potent urinary biomarker of kidney graft fibrosis and injury in other studies [13,14], was not associated with tubulointerstitial fibrosis in our study. 
We observed TG2 and SDC4 intensity upregulation on protocol biopsy 6-month posttransplant in the IFTA group. Both TG2 and SDC4 upregulation was remarkable especially in tubular structures. Colocalization of TG2 and HSPG was significant at the 6-month biopsy in the IFTA group. Localization of SDC4 within the nucleus was observed in a representative 6-month biopsy of the IFTA group, although confocal microscopy did not demonstrate a significant increase in MFI. Our results are consistent with previous in vitro and animal studies. We hypothesized that in patients with ongoing progression of tubulointerstitial fibrosis of the graft kidney during early posttransplantation period, TG2 expression would be upregulated and the extracellular TG2 level increases, hence, resulting in high levels of urinary TG2. Interaction between TG2 and SDC4 during development of allograft fibrosis leads to nuclear localization of HSPG and SDC4, where the role of HSPG in regulating nuclear function remains unclear. It is possible that the nuclear localization of SDC4 results in decreased extracellular SDC4 levels which are detected as reduced urinary SDC4 levels.

There are several limitations to this study. This is an observational study at a single institution evaluating a relatively small number of patients. Furthermore, a common challenge of protein analysis of spot urine samples collected involves differences in the abundance of each protein or metabolite in biological fluids and the concentration of biological fluids is affected by various factors and by the physiological status of the patients [29]. There are several cases with missing data of urinary biomarker levels which may have possibly influenced the small cohort sample present in our study. Repeated measurements in a larger patient population with a longer posttransplantation follow-up period may further support and strengthen our observations. However, we demonstrate for the first time in the DDKT population that kidney allograft fibrosis is associated with increased levels of urinary TG2 and, hence, we propose TG2 as a potential candidate as a promising urinary biomarker. Further studies are necessary to obtain causation confirmation and subsequent studies for multiple validation steps of performance evaluation and impact determination are required to appropriately develop TG2 as an efficient novel biomarker.

We suggest urinary TG2 as a potential biomarker to determine kidney graft fibrosis during the early postoperative period in DDKT recipients. Future studies to verify and confirm this relationship are necessary. Collecting urine for clinical measurements is less invasive than performing kidney biopsies and may facilitate repeating measurements in patients to determine risk and responses to treatment. Measurements of urinary TG2 may provide an opportunity to monitor kidney transplantation recipients serially and noninvasively and to identify those at higher risk of graft fibrosis, in whom closer surveillance and prevention treatments may be warranted.

\section{CONFLICTS OF INTEREST}

No potential conflict of interest relevant to this article was reported.

\section{ACKNOWLEDGEMENTS}

This research was performed with grant support from the Ministry of Education (2014R1A1A2A16055405) and Ministry of Science \& ICT (2016M3A9E8941330) of Republic of Korea.

\section{REFERENCES}

1. Joosten SA, Sijpkens YW, van Kooten C, Paul LC. Chronic renal allograft rejection: pathophysiologic considerations. Kidney Int 2005;68:1-13.

2. Williams WW, Taheri D, Tolkoff-Rubin N, Colvin RB. Clinical role of the renal transplant biopsy. Nat Rev Nephrol 2012;8:11021.

3. Furness PN, Taub N; Convergence of European Renal Transplant Pathology Assessment Procedures (CERTPAP) Project. International variation in the interpretation of renal transplant bio- psies: report of the CERTPAP Project. Kidney Int 2001;60:1998-2012.

4. Haas M, Sis B, Racusen LC, Solez K, Glotz D, Colvin RB, et al. Banff 2013 meeting report: inclusion of c4d-negative antibodymediated rejection and antibody-associated arterial lesions. Am J Transplant 2014;14:272-83.

5. Christians U, Klawitter J, Klawitter J. Biomarkers in transplantation--proteomics and metabolomics. Ther Drug Monit 2016;38 Suppl 1:S70-4.

6. Schaub S, Rush D, Wilkins J, Gibson IW,
Weiler T, Sangster K, et al. Proteomicbased detection of urine proteins associated with acute renal allograft rejection. J Am Soc Nephrol 2004;15:219-27.

7. Scarpellini A, Huang L, Burhan I, Schroeder N, Funck M, Johnson TS, et al. Syndecan-4 knockout leads to reduced extracellular transglutaminase-2 and protects against tubulointerstitial fibrosis. J Am Soc Nephrol 2014:25:1013-27.

8. Racca MA, Novoa PA, Rodríguez I, Della Vedova AB, Pellizas CG, Demarchi M, et al. Renal dysfunction and intragraft 
proMMP9 activity in renal transplant recipients with interstitial fibrosis and tubular atrophy. Transpl Int 2015;28:71-8.

9. Levey AS, Stevens LA, Schmid CH, Zhang YL, Castro AF 3rd, Feldman HI, et al. A new equation to estimate glomerular filtration rate. Ann Intern Med 2009;150: 604-12.

10. Ix JH, Biggs ML, Mukamal K, Djousse L, Siscovick D, Tracy R, et al. Urine collagen fragments and CKD progression-the cardiovascular health study. J Am Soc Nephrol 2015;26:2494-503.

11. Ix JH, Katz R, Bansal N, Foster M, Weiner $\mathrm{DE}$, Tracy $\mathrm{R}$, et al. Urine fibrosis markers and risk of allograft failure in kidney transplant recipients: a case-cohort ancillary study of the FAVORIT trial. Am J Kidney Dis 2017;69:410-9.

12. Reese PP, Hall IE, Weng FL, Schroppel B, Doshi MD, Hasz RD, et al. Associations between deceased-donor urine injury biomarkers and kidney transplant outcomes. J Am Soc Nephrol 2016;27:1534-43.

13. Teppo AM, Tornroth T, Honkanen E, Gronhagen-Riska C. Urinary amino-terminal propeptide of type III procollagen (PIIINP) as a marker of interstitial fibrosis in renal transplant recipients. Transplantation 2003;75:2113-9.

14. Teppo AM, Honkanen E, Finne P, Tornroth T, Gronhagen-Riska C. Increased urinary excretion of alpha1-microglobulin at 6 months after transplantation is associated with urinary excretion of transforming growth factor-betal and indicates poor long-term renal outcome. Transplantation 2004:78:719-24.

15. Ho J, Rush DN, Krokhin O, Antonovici M, Gao A, Bestland J, et al. Elevated urinary matrix metalloproteinase-7 detects underlying renal allograft inflammation and injury. Transplantation 2016;100:64854.

16. Hricik DE, Nickerson P, Formica RN, Poggio ED, Rush D, Newell KA, et al. Multicenter validation of urinary CXCL9 as a risk-stratifying biomarker for kidney transplant injury. Am J Transplant 2013; 13:2634-44.

17. Jones RA, Kotsakis P, Johnson TS, Chau DY, Ali S, Melino G, et al. Matrix changes induced by transglutaminase 2 lead to inhibition of angiogenesis and tumor growth. Cell Death Differ 2006;13:144253.

18. Balajthy Z, Csomos K, Vamosi G, Szanto A, Lanotte M, Fesus L. Tissue-transglutaminase contributes to neutrophil granulocyte differentiation and functions. Blood 2006;108:2045-54.

19. Stephens P, Grenard P, Aeschlimann P, Langley M, Blain E, Errington R, et al. Crosslinking and G-protein functions of transglutaminase 2 contribute differentially to fibroblast wound healing responses. J Cell Sci 2004:117(Pt 15):3389-403.

20. Johnson TS, El-Koraie AF, Skill NJ, Baddour NM, El Nahas AM, Njloma M, et al. Tissue transglutaminase and the progression of human renal scarring. J Am Soc Nephrol 2003;14:2052-62.

21. Verderio EA, Johnson TS, Griffin M. Transglutaminases in wound healing and inflammation. Prog Exp Tumor Res 2005; 38:89-114.

22. Oh K, Park HB, Byoun OJ, Shin DM, Jeong EM, Kim YW, et al. Epithelial transglutaminase 2 is needed for $\mathrm{T}$ cell interleukin-17 production and subsequent pulmonary inflammation and fibrosis in bleomycin-treated mice. J Exp Med 2011; 208:1707-19.

23. Shweke N, Boulos N, Jouanneau C,
Vandermeersch S, Melino G, Dussaule JC, et al. Tissue transglutaminase contributes to interstitial renal fibrosis by favoring accumulation of fibrillar collagen through TGF-beta activation and cell infiltration. Am J Pathol 2008;173:631-42.

24. Shin S, Cho YP, Jun H, Park H, Hong HN, Kwon TW. Transglutaminase type 2 in human abdominal aortic aneurysm is a potential factor in the stabilization of extracellular matrix. J Vasc Surg 2013:57: 1362-70.

25. Scarpellini A, Germack R, Lortat-Jacob H, Muramatsu T, Billett E, Johnson T, et al. Heparan sulfate proteoglycans are receptors for the cell-surface trafficking and biological activity of transglutaminase-2. J Biol Chem 2009;284:18411-23.

26. Belkin AM. Extracellular TG2: emerging functions and regulation. FEBS J 2011;278: 4704-16.

27. Richardson TP, Trinkaus-Randall V, Nugent MA. Regulation of heparan sulfate proteoglycan nuclear localization by fibronectin. J Cell Sci 2001;114(Pt 9):1613-23.

28. Wee YM, Lee HW, Choi MY, Jung HR, Choi JY, Kwon HW, et al. A composite of urinary biomarkers for differentiating between tubulointerstitial inflammation and interstitial fibrosis/tubular atrophy in kidney allografts. Ann Hepatobiliary Pancreat Surg 2018;22:310-20.

29. Price JW. Creatinine normalization of workplace urine drug tests: does it make a difference? J Addict Med 2013;7:129-32.

30. Zhao F, Song S, Liu W, Keep RF, Xi G, Hua Y. Red blood cell lysis and brain tissuetype transglutaminase upregulation in a hippocampal model of intracerebral hemorrhage. Acta Neurochir Suppl 2011; 111:101-5. 